\title{
Intervenciones para reducir el consumo de sal a través del etiquetado
}

\author{
Javier Sanz-Valero, ${ }^{1}$ Miren Itxaso Sebastián-Ponce ${ }^{1}$ \\ y Carmina Wanden-Berghe ${ }^{2}$
}

Forma de citar

Sanz-Valero J, Sebastián-Ponce MI, Wanden-Berghe C. Intervenciones para reducir el consumo de sal a través del etiquetado. Rev Panam Salud Publica. 2012;31(4)332-7.

RESUMEN Objetivo. Determinar el grado en que el etiquetado de productos alimentarios informa acerca del consumo de sal.

Métodos. Se realizó un análisis crítico y sistemático de 9 estudios —seleccionados de un total de 133 - recogidos mediante revisión de la literatura científica sobre las intervenciones realizadas en población humana orientadas a reducir el consumo de sal a través de mensajes en el etiquetado. Toda la información se obtuvo mediante consulta directa y vía Internet a la literatura científica recogida en varias bases de datos.

Resultados. De los 133 artículos recuperados, una vez aplicados los criterios de inclusión y exclusión, se seleccionaron para la revisión 9 trabajos: en todos ellos se planteaba a la población en estudio su conocimiento acerca de la interpretación de la etiqueta sobre el contenido de sal de los alimentos.

Conclusiones. Los consumidores de alimentos entienden y valoran más a los logotipos que a la composición nutricional que figura en la etiqueta. Se justificaría entonces el uso de logotipos alternativos que facilitaran esta información y que además fueran normalizados. Esta situación se ve reforzada porque la inclusión de símbolos fácilmente entendibles favorece la correcta elección por parte de los consumidores.

Palabras clave Etiquetado de alimentos; sodio; sodio en la dieta; seguridad alimentaria; promoción de la salud; política de salud.

La sal es quizá el condimento más antiguo usado por el hombre y su importancia para la vida ha sido tal que ha tenido grandes repercusiones económicas, políticas y culinarias a lo largo de la historia (1). Utilizada para la conservación de alimentos durante siglos, su importancia económica fue reduciéndose a medida que la industria contaba con métodos

\footnotetext{
1 Universidad de Alicante, Departamento de Enfermería Comunitaria, Medicina Preventiva y Salud Pública e Historia de la Ciencia, Alicante, España. La correspondencia se debe dirigir a Javier SanzValero. Correo electrónico: javier.sanz@ua.es

2 Universidad Cardenal Herrera CEU, Departamento de Fisiología, Farmacología y Toxicología, Elche, España.
}

de conservación alimentaria y técnicas de extracción y elaboración de sal más avanzados y efectivos. Sin embargo, pese a su declinante protagonismo en la historia, la sal continúa siendo un ingrediente muy común y a veces imprescindible en cualquier cocina de hoy (2).

La otra cara de la moneda es que hay un constante interés en reducir el consumo de sodio porque se lo asocia con varios problemas de salud que incluyen hipertensión, daño vascular y cardiaco, obesidad, cáncer de estómago, osteoporosis, cálculos renales e incremento de la severidad de los síntomas del asma (3). Alrededor de $80 \%$ de la sal que se ingiere proviene de alimentos procesados, entre ellos el relevante caso del pan, que aporta (4) una sexta parte de la sal que consume diariamente el mundo desarrollado (5). En este sentido, se ha demostrado que reducir la sal en el pan una cuarta parte no produce diferencias respecto a sus características organolépticas (6). Un ejemplo se ha dado en Nueva Zelanda, donde la reformulación de ciertos productos industriales (p. ej. panes, cereales de desayuno y margarinas) en un año disminuyó el consumo de sal en 33 toneladas (7).

Según la Organización Mundial de la Salud, los gobiernos de todo el mundo 
podrían ahorrar costos en salud y evitar millones de muertes prematuras si introdujeran normas para bajar los niveles de sal en la comida, con un consumo máximo de $2000 \mathrm{mg}$ de sodio por día (8). Los gobiernos pueden intentar disminuir la ingesta de sodio de la población mediante legislación, normas y regulaciones, y aun desalentando su consumo mediante mensajes en el etiquetado alimentario. No obstante, sin campañas de información y de educación al público idóneas, estos esfuerzos no han dado muy buenos resultados.

En ausencia de una adecuada educación, una clara y visible información nutricional explícita es útil. Con la correcta información y educación, las medidas legales toman vida fácilmente para los que quieren reducir su ingesta de sal (9). En España, la Estrategia para la Nutrición, Actividad Física y Prevención de la Obesidad (NAOS) observa un plan de reducción del consumo de sal basado en la educación, la reformulación, la recopilación de datos, la información, y el seguimiento y la evaluación de las medidas llevadas a cabo (10). En este marco, el objetivo del presente trabajo es determinar el grado en que el etiquetado de productos alimentarios informa acerca del consumo de sal.

\section{MATERIALES Y MÉTODOS}

Se realizó un análisis crítico y sistemático de los trabajos recogidos mediante revisión de la literatura científica sobre las intervenciones realizadas en población humana orientadas a reducir el consumo de sal a través de mensajes en el etiquetado. Toda la información se obtuvo mediante consulta directa y vía Internet a la literatura científica recogida en las bases de datos Medlars Online International Literature (MEDLINE), vía PubMed ${ }^{\odot}$; EMBASE$^{\odot} ;$ Web of Knowledge (Institute for Scientific Information); The Cochrane Library Plus, Food Science and Technology Abstracts (FSTA); Latin American and Caribbean Health Sciences Literature (LILACS); y The Cumulative Index to Nursing and Allied Health Literature (CINAHL).

Se estudiaron los artículos publicados en cualquier país, por cualquier autor (corporativo o individual) y en cualquier idioma, publicados desde el inicio de la indización de cada una de las fuentes primarias. Para la recuperación documental se emplearon los descriptores (Medical
Subject Headings, MeSH) desarrollados por la Biblioteca Nacional de Medicina (Estados Unidos). No se utilizaron calificadores de materia (subheadings), ni fue necesario el empleo de tags. Del estudio del Thesaurus se consideraron adecuados los descriptores "sodium", "sodium, dietary", "food labelling", "health promotion" y "health policy". Se utilizó el límite "humanos".

Las ecuaciones de búsqueda se desarrollaron para emplearlas en la base de datos MEDLINE, vía PubMed, mediante la utilización de los conectores booleanos, adaptándolas luego a las bases de datos recién mencionadas. La búsqueda se efectuó desde la primera fecha disponible, de acuerdo a las características de cada una de las bases de datos, hasta diciembre de 2010 — momento de la última actualización. La elección final de los artículos incluyó a todos los documentos originales publicados en revistas científicas arbitradas, incluyendo aquellos artículos pertinentes cuyo texto pudo ser recuperado. Se excluyeron los trabajos que no versaban sobre etiquetado, así como los que no habían sido estudiados en una población. Además, como búsqueda secundaria y para reducir posibles sesgos de publicación, se examinó el listado bibliográfico de los artículos que fueron seleccionados en la búsqueda principal con el objeto de identificar estudios no detectados en la revisión electrónica.

Dos de los autores del presente trabajo evaluaron separadamente la pertinencia de los artículos. Para dar por válida la elección de los artículos seleccionados se estableció que la valoración de la concordancia entre estos dos autores (índice Kappa) debía ser superior a 0,60 (fuerza de la concordancia buena o muy buena). Siempre que se cumpliera esta condición, las posibles discordancias se solucionarían mediante consulta a un tercer autor y posterior consenso entre todos los autores.

El control de calidad de la información se realizó mediante tablas de doble entrada que permitían detectar y corregir errores mediante nueva consulta con los originales. Los trabajos escogidos fueron agrupados con objeto de sistematizar -y facilitar - la comprensión de todos los resultados de la muestra. Ningún artículo fue rechazado por causas metodológicas. No se establecieron restricciones en cuanto a la edad o el género de los participantes, ni tampoco acerca del tipo de muestra.
Todos los datos relevantes de cada trabajo se resumieron en una tabla, codificándolos según autores, año de publicación, intervención efectuada, la población sometida a estudio, lugar donde se efectuó la intervención y resultados.

\section{RESULTADOS}

De los 133 artículos recolectados en un inicio, $62(46,61 \%)$ fueron de EMBASE, 46 (34,58\%) de MEDLINE, 9 $(6,76 \%)$ de CINAHL, 13 (9,77\%) de Web of Knowledge (Institute for Scientific Information) y $3(2,25 \%)$ de Food Science and Technology Abstracts. No se obtuvieron resultados en la base de datos bibliográficos LILACS. Una vez aplicados los criterios de inclusión y exclusión, se seleccionaron nueve artículos (11-19) (ver cuadro 1). No fue necesario valorar la concordancia entre los autores porque el acuerdo sobre pertinencia fue de $100 \%$.

De los nueve trabajos seleccionados, cuatro $(44,4 \%)$ se realizaron en Estados Unidos $(11,16,18,19)$, dos $(22,2 \%)$ en Australia $(12,13)$, uno $(11,1 \%)$ en Finlandia (14), uno $(11,1 \%)$ en Nueva Zelanda (15) y uno $(11,1 \%)$ en Reino Unido (16). En Estados Unidos se publicó el artículo más actual -2010- (11) y también el más antiguo -1984 (19).

La información encontrada sobre la población sujeta a intervención fue muy heterogénea. Algunos estudios aportaron datos muy precisos sobre edad, género, etnia, nivel cultural e ingresos, mientras que otros no aportaron información sobre estos temas $(11,14,17)$. La intervención con mayor número de participantes tuvo 4008 y la de menor número tuvo $50(16,18)$. Si bien los estudios no seleccionaron la población por género, en todas las intervenciones -excepto en la hospitalaria- el número de mujeres fue superior al de los hombres (18).

La encuesta/entrevista/cuestionario fue el método utilizado para recabar información, utilizándose varias modalidades: dos por correo postal $(11,17)$, dos por teléfono $(12,13)$, dos mediante entrevista en la calle $(16,19)$ y tres mediante llenado de un cuestionario (14, 15, 18). Los lugares donde se llevaron a cabo las consultas fueron principalmente los hogares $(11,14,16,17,19)$, centros comerciales $(12,13)$, un hospital (18) y otra localización no especificada en el trabajo revisado (15).

El diseño de todas las intervenciones fue transversal, aunque para el estudio 
efectuado en el hospital se realizó un seguimiento sobre el consumo de sodio a los 5 meses (17).

En cuanto a los resultados observados en los trabajos examinados, destacan el interés y la preocupación de los individuos de las muestras por el contenido de sal en los alimentos (11-19). En su mayoría, la población desconocía la cantidad de sal diaria que era recomendable consumir y no comprendía la información nutricional que figuraba en el etiquetado $(12,13,15,16,18)$. En este sentido, aun sin ser capaces de descifrar los datos, todos los encuestados sabían que el etiquetado informaba sobre el contenido de sal en los productos $(12,15)$. Por ejemplo, la mitad de los participantes, tras leer las indicaciones de las etiquetas, no pudieron relacionar opciones de compra bajas en sodio, no conocían la relación entre los términos "sal" y "sodio" y subestimaron el contenido de sal $(12,16)$.

En uno de los artículos analizados, apenas 3\% de una muestra de 2400 personas adultas reconocía consultar el etiquetado nutricional de los productos (19). Las po-

\section{CUADRO 1. Características de los nueve estudios (evaluados) sobre intervenciones para reducir el consumo de sal a través del etiquetado}

\begin{tabular}{|c|c|c|c|c|}
\hline Autor, año & Intervención & Población (resumen) & Lugar & Resultado principal \\
\hline $\begin{array}{l}\text { Ayala et al., } \\
2010(11)\end{array}$ & $\begin{array}{l}\text { Encuesta anual por } \\
\text { correo, comparando } \\
\text { los años } 2005 \text { y } \\
2008\end{array}$ & 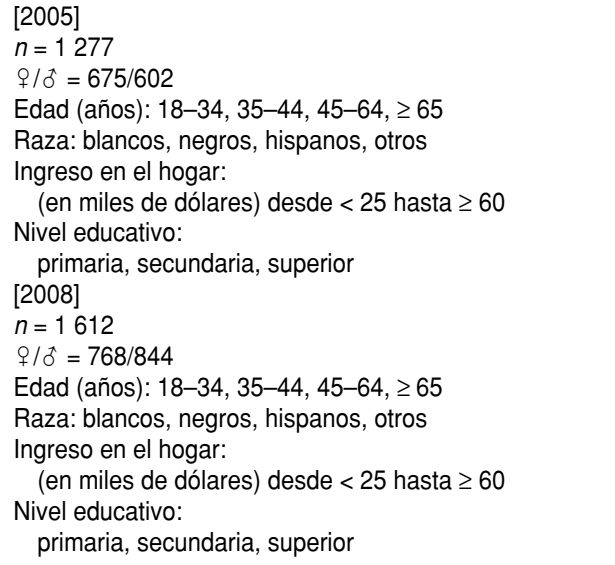 & Estados Unidos & $\begin{array}{l}\text { - Se redujo el consumo de sal de } 48 \% \text { a } 56 \% \\
\text { en } 2005-2008 \text {. } \\
\text { - Aumentó la lectura del contenido de las } \\
\text { etiquetas (y más las personas mayores, las } \\
\text { mujeres, las personas negras, las personas } \\
\text { con menores ingresos y con una educación } \\
\text { media) de } 64 \% \text { a } 69 \% \text {. }\end{array}$ \\
\hline $\begin{array}{l}\text { Grimes et al., } \\
2009 \text { (12) }\end{array}$ & Cuestionario & $\begin{array}{l}n=474 \\
\text { } / \text { } 0+=310 / 164 \\
\text { Edad (años): } 18-24,25-34,35-44,45-54,55-64, \geq 65 \\
\text { Etnia: caucásico, asiático y otros } \\
\text { Nivel educativo: } \\
\quad \text { primaria, secundaria, superior } \\
\text { Principal comprador del hogar } \\
\text { Sí/No = } 303 / 49 \\
\text { Comparte responsabilidad: } 122\end{array}$ & $\begin{array}{l}\text { Melbourne, } \\
\text { Australia } \\
\text { (centros } \\
\text { comerciales) }\end{array}$ & $\begin{array}{l}\text { - } 65 \% \text { fueron capaces de identificar la } \\
\text { relación entre sal y sodio. } \\
\text { - } 69 \% \text { dijo leer el etiquetado de la sal a la } \\
\text { hora de la compra. } \\
\text { - El uso de la etiqueta de sal se relacionó } \\
\text { significativamente con la preocupación } \\
\text { de los compradores acerca de la } \\
\text { cantidad de sal en su dieta y la creencia } \\
\text { de que su salud podría mejorar mediante } \\
\text { la reducción de la ingesta de sal. } \\
\text { - } 50 \% \text { de la muestra fue incapaz de } \\
\text { realizar opciones de compra bajas en sal } \\
\text { a pesar de apoyarse en la etiqueta. }\end{array}$ \\
\hline $\begin{array}{l}\text { Kelly et al., } \\
2009 \text { (13) }\end{array}$ & $\begin{array}{l}\text { Cuestionario para } \\
\text { determinar cuál } \\
\text { de los etiquetados } \\
\text { existentes en la } \\
\text { parte frontal es el } \\
\text { más entendible y } \\
\text { preferido por los } \\
\text { entrevistados }\end{array}$ & $\begin{array}{l}n=790 \\
\text { } / \delta=538 / 252 \\
\text { Edad (años): } 18-19,20-29,30-39,40-49,50-59 \\
\quad 60-69,70-79, \geq 80 \\
\text { Responsabilidad en las compras: compartido/principal } \\
\text { Composición del hogar: } \\
\quad \text { (Vive solo, compañero/ pareja/esposo, con niños, } \\
\text { con adolescentes) } \\
\text { Nivel educativo: primaria, secundaria, superior } \\
\text { Ingresos en el hogar: } \\
\text { (en miles de dólares) desde < } 30 \text { hasta } \geq 90 \\
\text { País de nacimiento: Australia u otro }\end{array}$ & $\begin{array}{l}\text { Nueva Gales del } \\
\text { Sur, Australia }\end{array}$ & $\begin{array}{l}\text { Utilizando el traffic light (semáforo de } \\
\text { advertencia), fue cinco veces más probable } \\
\text { que los entrevistados identificaran productos } \\
\text { más saludables que con otra de las etiquetas } \\
\text { monocromas, y tres veces más probable que } \\
\text { con la etiqueta de código de color. }\end{array}$ \\
\hline $\begin{array}{l}\text { Mackinson et al., } \\
2009(14)\end{array}$ & $\begin{array}{l}\text { Encuesta por } \\
\text { correo a diferentes } \\
\text { cadenas de } \\
\text { restaurantes en } \\
\text { el Reino Unido } \\
\text { (frecuencia de sus } \\
\text { comidas, deseo } \\
\text { de información } \\
\text { nutricional ...) }\end{array}$ & $\begin{array}{l}n=784 \\
\$ / \widehat{ }=506 / 278 \\
\text { Edad (años): } 18-25,26-35,36-49,50-65, \geq 66 \\
\text { Estatus: ricos, acomodados, clase media, clase } \\
\quad \text { media-baja, desfavorecidos } \\
\text { Motivación "elegir una dieta saludable": } \\
\quad \text { extremado, moderado, poco, nada motivado } \\
\text { Lugar de residencia: Inglaterra, Escocia, Gales, } \\
\quad \text { Irlanda del Norte }\end{array}$ & Reino Unido & $\begin{array}{l}\text { - } 65 \% \text { dijo estar "motivado a comer una } \\
\text { dieta saludable". } \\
\text { - } 40 \% \text { informó comer en un restaurante de } \\
\text { comida de catering > } 1 \text { vez a la semana. } \\
\text { - > } 50 \% \text { dijeron que deseaban ver } \\
\text { información sobre los ingredientes y el } \\
\text { contenido de sal de elementos de menú, } \\
42-56 \% \text {, la energía y } 47-59 \% \text {, la grasa. } \\
43 \% \text { de los encuestados agradecería } \\
\text { recibir información sobre el contenido } \\
\text { energético en menú de restaurantes. }\end{array}$ \\
\hline
\end{tabular}


CUADRO 1. (Continuación)

\begin{tabular}{ll}
\multicolumn{1}{c}{ Autor, año } & \multicolumn{1}{c}{ Intervención } \\
\hline $\begin{array}{l}\text { Pietinen et al., } \\
2007 \text { (15) }\end{array}$ & $\begin{array}{l}\text { Encuesta nacional } \\
\text { FINDIET 2002 con } \\
\text { recuerdos de 48 } \\
\text { horas de 2 } 007 \\
\text { personas }\end{array}$ \\
& \\
Gilbey et al., & $\begin{array}{l}\text { Cuestionario de } \\
\text { preguntas cortas } \\
\text { sobre la conciencia } \\
\end{array}$ \\
& $\begin{array}{l}\text { que tienen sobre } \\
\text { su ingesta de sal } \\
\text { y la estimación del } \\
\text { contenido de sal } \\
\text { en productos con } \\
\text { etiqueta }\end{array}$
\end{tabular}

$\begin{array}{ll}\text { Neily et al, } & \text { Tests realizados } \\ 2002(17) & \text { por la dietista antes } \\ & \text { y después de la } \\ & \text { formación de las } \\ & \text { pacientes }\end{array}$

Heimbach 1985 (18)

\author{
Encuesta telefónica \\ realizada utilizando \\ un método de \\ marcado aleatorio \\ de dígitos en \\ 1984 (no ofrece \\ datos sobre los \\ encuestados, solo \\ porcentajes de los \\ resultados)
}

Población (resumen)

$n=2007$

$q /$ đ $=1095 / 912$

Edad (años): 25-64

o $\mu=45(\mathrm{DE} 11,6)$

oे $\mu=46$ (DE 11,3) Nivel educativo (en años):

o $\mu=11.3$ (DE 3,8)

๙ิ $\mu=11.6$ (DE 3,8)

$n=226$

९/ठ = 139/87

Edad (años): $<20,21-30,31-40,41-50,51-60, \geq 61$

$n=50$

$\delta / 9=25 / 25$

Edad (años): $53 \pm 10$

Etnia: afroamericano, blanco, hispano

Todos ellos con insuficiencia cardiaca crónica (ICC)

$n=4008$

Edad (años): > 18

Etnia: habla inglesa e hispana
Lugar

Resultado principal

Finlandia

La media de sal consumida se reduciría en $1,8 \mathrm{~g}$ en hombres y en $1,0 \mathrm{~g}$ en mujeres, si la población eligiera los productos bajos en sal; y si además se redujera a la mitad la sal añadida en las comidas caseras, se reduciría en $2,5 \mathrm{~g}$ y $1,8 \mathrm{~g}$, respectivamente.

Nueva Zelanda La mayoría de los participantes no supieron interpretar la información nutricional y subestimaron el contenido de sal, confundiéndolo con el contenido de sodio (solo cuatro de los participantes lo realizan de forma adecuada).

Tampoco saben qué cantidad de sal deben consumir en un día (solo 10,2\% lo sabía).

$1000 \mathrm{mg}$ sodio $\approx 2500 \mathrm{mg}$ sal (se puede subestimar en 2,5 si se piensa que es lo mismo).

Este estudio sugiere que la población en Nueva Zelanda no entiende la información en el etiquetado, por lo que es de poca utilidad en su forma actual.

Dallas, Texas, En referencia a la clínica para ICC, muchos mayo de 1999agosto de 2000 (hospital) pacientes tenían deficiencias graves en su conocimiento con respecto a la ingesta de sodio en la dieta, y se han corregido muchas de estas deficiencias después de la intervención. El seguimiento confirma que estos pacientes, adquiridas las habilidades, las continúan aplicando.

Estados Unidos $\quad 38 \%$ de los encuestados dijeron leer la etiqueta del producto para evitar el exceso de sal. La causa de que las personas no se interesen en productos bajos en sal son:

- $49 \%$ opina que son más caros que los de siempre.

- $38 \%$ opina que no tienen buen sabor.

- 35\% piensa que va dirigido a población con problemas médicos.

- Solo $30 \%$ de las personas preocupadas por la sal en los productos encuentra la información útil y entendible (en 1982 fueron 35\%).

- $57 \%$ pensó que las palabras sal y sodio eran intercambiables.

Heimbach et al., Entrevista telefónica $n=2400$ 1984 (19) con marcado $\quad$ Edad = adultos aleatorio de dígitos llevada a cabo en 1982 sodio, y otro $49 \%$ indicó un cierto grado
Estados Unidos - $\quad$ - 12\% de los encuestados había sido aconsejado por un profesional de la salud para reducir el consumo de de preocupación —desde el deseo ocasional de usar menos sal a dietas estrictas bajas en sodio autoimpuesta.

- $61 \%$ de los encuestados fueron caracterizados "preocupados por el sodio", y la mayoría de preguntas sobre el etiquetado de sodio se dirigieron solo a ellos.

- De $23 \%$ que dijo haber sido prescrito una dieta baja en sodio, solo la mitad recordó algún consejo específico. El $10 \%$ dijo buscar etiquetas "bajas en sal", $5 \%$ mirar la lista de ingredientes, y $3 \%$ el etiquetado nutricional.

Fuente: elaboración de los autores.

Nota: DE (desviación estándar). 
blaciones encuestadas revelaron la creencia de que los productos con bajo contenido en sal son más caros y por eso no son consumidos (18). Dos artículos trabajaron la identificación correcta de los símbolos por parte de la población entrevistada, indicando que un código de color que identifique claramente el contenido de sal en las etiquetas de los productos podría favorecer una solución $(12,13)$. En cuanto al consumo de sal en los alimentos de restaurantes, más de la mitad de los consultados dijeron que agradecerían recibir información al respecto (14). En el único estudio llevado a cabo en el hospital se comprobó que, tras adquirir las habilidades para seleccionar alimentos con menos sal, esta práctica seguía aplicándose varios meses después (17).

\section{DISCUSIÓN}

Si bien la lectura de la etiqueta es la forma más directa y simple que el consumidor tiene de conocer el contenido en sal de los productos alimentarios, se encontró que la gran mayoría de las personas encuestadas desconocían la cantidad máxima recomendada de sal que debían consumir al día y gran parte ignoraban la diferencia entre los términos "sal" y "sodio". Esta realidad por sí misma justificaría el uso de logotipos alternativos que faciliten al consumidor esta información y, mejor aún, si estos logotipos estuvieran normalizados y fueran fáciles de identificar por la población (20).

De hecho, se ha probado la efectividad que tienen las etiquetas con símbolos saludables, además de visibles y "amigables", para transmitir el mensaje a los consumidores $(13,21,22)$. Laethwood y colaboradores y Hooker y Teratanavat llegan a la conclusión de que los consumidores entienden y valoran mejor estos logotipos a la hora de tomar decisiones saludables de compra, reforzando la opinión de otros dos estudios de que la inclusión de símbolos fácilmente entendibles favorece la correcta elección por parte de los consumidores (12, 13, 23, 24).

Contrariamente, se hallaron casos en que las estrategias para reducir el consumo de sal basadas en el etiquetado no son efectivas porque los logotipos que identifican a los productos bajos en sal no son reconocidos o no son entendidos por parte de la población o su mensaje no llega con claridad $(20,21,25)$. Se halló también que los intentos de promover un "consumidor informado" con la esperanza de que pueda elegir los alimentos de forma más saludable no han logrado el éxito esperado, planteándose así el difícil desafío de conseguir cambios en el comportamiento de los consumidores (26). Cabe destacar la relevancia que tienen el apoyo de los medios de comunicación y las escuelas como instrumentos para educar acerca los efectos de la sal y la difusión de los logotipos.

En este contexto, y dado que la sal forma parte de muchos alimentos altamente consumidos, una opción efectiva para su reducción podría ser la reformulación de los productos siempre y cuando no se llegue a alterar sus características organolépticas —un ejemplo es el ya mencionado programa en Nueva Zelanda (7). Reformular alimentos procesados con sodio debería ser una medida efectiva, sobre todo si se aplica a los productos que contienen entre $75 \%$ y $80 \%$ de la sal que se consume, y no supondría costos de elaboración adicionales (27). Igualmente, sería oportuno estudiar la importancia del consumo de aguas bicarbonatadas -y su aportación de sodio a la dieta- particularmente en poblaciones con patologías específicas que son afectadas de forma negativa por el sodio (28). Una investigación reciente reafirma el beneficio para la salud de disminuir o aun limitar el contenido de sal en los alimentos (29). Una mínima reducción en la ingesta de sal puede ser tan ventajosa, en términos de salud pública, como dejar de fumar (30).

Otro de los problemas observados en los estudios fue la dificultad de conocer la cantidad de sal que contienen los productos consumidos en restaurantes y de catering, donde se aprovecha la sal para realzar el sabor de los alimentos (31). Como se ha visto, en una sociedad en la que comer fuera de casa o consumir alimentos precocinados son opciones habituales, esta situación es de alto interés (17). Las sensaciones, sobre todo sabor y aroma, tienen mucho que ver en las decisiones de consumo; se sabe que en los tan frecuentados restaurantes de comida rápida el atractivo sabor de los alimentos se logra en gran medida mediante un alto contenido en sal (32-35).

Los autores reconocen que hubiera sido preferible contar con una muestra de estudios que incluyeran períodos de seguimiento adecuados y diseños que garanticen la evidencia científica de las conclusiones. Como se ha dicho, se decidió incluir todos los trabajos recuperados y considerados como pertinentes, con la única condición de haber estudiado la actuación de las personas en relación al contenido de sal manifestada en el etiquetado.

Finalmente, el análisis permite concluir que los consumidores de alimentos entienden y valoran más a los logotipos que a la composición nutricional que figura en la etiqueta. Se justificaría entonces el uso de logotipos alternativos que facilitaran esta información y que además fueran normalizados. Esta situación se ve reforzada porque la inclusión de símbolos fácilmente entendibles favorece la correcta elección por parte de los consumidores.

\section{REFERENCIAS}

1. Instituto de la Sal. Historia de la sal. 2011. Disponible en: http://www.institutodelasal. com/index.php?page=hist Acceso el 12 de febrero de 2012.

2. Harvard Medical School. Salt and your health, part II: shaking the habit. Harv Mens Health Watch. 2003;8(1):1-4.

3. Mohan S, Campbell N, Willis K. Effective population-wide public health interven- tions to promote sodium reduction. CMAJ. 2009;181(9):605-9.

4. Council on Scientific Affairs. Sodium in processed foods. JAMA. 1983;249(6): 784-9.

5. Sharp D. Labelling salt in food: if yes, how? Lancet. 2004;364(9451):2079-81.

6. Levy LB. Food policy and dietary change. Proc Nutr Soc. 2009;68(2):216-20.
7. Young L, Swinburn B. Impact of the Pick the Tick food information programme on the salt content of food in New Zealand. Health Promot Int. 2002;17(1):13-9.

8. Organización Mundial de la Salud. Reducción del consumo de sal en la población: informe de un foro y una reunión técnica de la OMS [monografía en Internet]. París, Francia: OMS; 2007. Disponible en: http://www.who.int/ 
dietphysicalactivity/salt-report-SP.pdf Acceso el 12 de febrero de 2012

9. Bruce A. Sodium in nutrition policy. Ann Clin Res. 1984;16(43):174-8.

10. Agencia Española de Seguridad Alimentaria. Estrategia NAOS. Disponible en: http:// www.naos.aesan.msps.es/naos/ficheros/ estrategia/estrategianaos Acceso el 12 de febrero de 2012

11. Ayala C, Tong X, Valderrama A, Ivy A, Keenan $\mathrm{N}$. Actions taken to reduce sodium intake among adults with self-reported hypertension: Health Styles survey, 2005 and 2008. J Clin Hypertens (Greenwich). 2010;12(10):793-9.

12. Grimes CA, Riddell LJ, Nowson CA. Consumer knowledge and attitudes to salt intake and labelled salt information. Appetite. 2009;53(2):189-94

13. Kelly B, Hughes C, Chapman K, Louie J CY, Dixon H, Crawford J, et al. Consumer testing of the acceptability and effectiveness of front-of-pack food labelling systems for the Australian grocery market. Health Promot Int. 2009;24(2):120-9.

14. Mackinson D, Wrieden WL, Anderson AS. Making an informed choice in the catering environment: what do consumers want to know? J Hum Nutr Diet. 2009;22(6):567-73.

15. Pietinen $P$, Valsta LM, Hirvonen T, Sinkko $H$. Labelling the salt content in foods: a useful tool in reducing sodium intake in Finland. Public Health Nutr. 2008;11(4):335-40.

16. Gilbey A, Fifield S. Nutritional information about sodium: is worth its salt? N Z Med J. 2006;119(1232):U1934.

17. Neily JB, Toto KH, Gardner EB, Rame JE, Yancy CW, Sheffield MA, et al. Potential contributing factors to noncompliance with dietary sodium restriction in patients with heart failure. Am Heart J. 2002;143(1):29-33.
18. Heimbach JT. Sodium, hypertension and the American public: second tracking survey. Public Health Rep. 1985;100(4):371-2.

19. Heimbach JT, Orwin RG. Public perceptions of sodium labelling. J Am Diet Assoc. 1984;84(10):1217-9.

20. Gilbert PA, Heiser G. Salt and health: the CASH and BPA perspective. Nutr Bull. 2005;30(1):62-9.

21. Colby SE, Johnson L, Scheett A, Hoverson B. Nutrition Marketing on Food Labels. J Nutr Educ Behav. 2010;42(2):92-8.

22. Montero Marín A, Limia Sánchez A, Franco Vargas E, Belmonte Cortés S. Estudio de declaraciones nutricionales y saludables en el etiquetado de leches fermentadas. Nutr Hosp. 2006;21(3):338-45.

23. Laethwood PD, Richardson DP, Sträter P, Todd PM, van Trijp HC. Consumer understanding of nutrition and health claims: sources of evidence. Br J Nutr. 2007;98(3):474-84.

24. Hooker NH, Teratanavat R. Dissecting qualified health claims: evidence from experimental studies. Crit Rev Food Sci Nutr. 2008;48(2):160-76.

25. Jain A, Sherman SN, Chamberlin LA, Carter Y, Powers SW, Whitaker RC. Why don't low-income mothers worry about their preschoolers being overweight? Pediatrics. 2001; 107(5):1138-46.

26. Borgmeier I, Westenhoefer J. Impact of different food label formats on healthiness evaluation and food choice of consumers: a randomized-controlled study. BMC Public Health. 2009;9:184.

27. Kaplan NM. The dietary guideline for sodium: should we shake it up? No. Am J Clin Nutr. 2000;71(5):1020-6.

28. Schoppen S, Sánchez-Muniz FJ, PérezGranados AM, Gómez-Gerique JA, Sarriá B,
Navas-Carretero $S$, et al. ¿Modifica el agua mineral bicarbonatada rica en sodio la sensibilidad a la insulina de las mujeres postmenopáusicas? Nutr Hosp. 2007;22(5):538-44.

29. Legowski B, Legetic B. How three countries in the Americas are fortifying dietary salt reduction: a north and south perspective. Health Policy. 2011;102(1):26-33.

30. He FJ, Jenner KH, MacGregor GA. WASHWorld action on SALT and health. Kidney Int 2010;78(8):745-53.

31. Chisholm A, Mann J. Reducing salt intake: action beyond the label. N Z Med J. 2006;119(1232):U1937.

32. Elliot C. Assessing "fun foods": nutritional content and analysis of supermarket foods targeted at children. Obes Rev. 2008;9(4):36877.

33. Hayne CL, Moran PA, Ford MM. Regulating environments to reduce obesity. J Public Health Policy. 2004;25(3-4):391-407.

34. Rydell SA, Harnack LJ, Oakes JM, Story M, Jeffery RW, French SA. Why eat at fastfood restaurants: reported reasons among frequent consumers. J Am Diet Assoc. 2008;108(12):2066-70.

35. O'Dougherty M, Harnack LJ, French SA, Story M, Oakes JM, Jeffery RW. Nutrition labeling and value size pricing at fast-food restaurants: a consumer perspective. Am J Health Promot. 2006; 20(4):247-50.

Manuscrito recibido el 24 de julio de 2011. Aceptado para publicación, tras revisión, el 22 de diciembre de 2011.

ABSTRACT Objective. Determine the extent to which labeling of food products informs about salt consumption.

Methods. A critical and systematic analysis was conducted of 9 studies selected out

Interventions to reduce salt consumption through labeling of a total of 133 studies. The studies were collected by reviewing the scientific literature on interventions conducted in the human population aimed towards reducing salt consumption through label messaging. All of the information was obtained by direct consultation and by Internet from the scientific literature collected in several databases.

Results. Out of the 133 articles recovered, after the inclusion and exclusion criteria were applied, 9 studies were selected for review. All of them took into account the ability of the study population to interpret and understand salt content labeling in foods.

Conclusions. Food consumers understand and value easily recognizable logos more than the information found on nutritional composition labels. Therefore, use of alternative logos that facilitate this information and are also standardized could be justified. This situation is reinforced because the inclusion of symbols that are easily understandable favors the most adequate choice by consumers.

Key words Food labeling; sodium; sodium, dietary; food security; health promotion; health policy. 